\title{
Resultados funcionales y radiográficos de la prótesis reversa y la reinserción del troquíter en pacientes con fracturas de húmero proximal
}

\author{
Juan Martín Patiño, Carlos E. Martínez, Bernardo Andres, Francisco López Bustos, César Ruiz, Hernán Fiminella, \\ Julio Ferro, Alberto Garay \\ Equipo de Cirugía de Mano y Miembro Superior, Instituto Dupuytren, Ciudad Autónoma de Buenos Aires, Argentina
}

\begin{abstract}
RESUMEN
Objetivo: Comunicar los resultados funcionales y radiográficos de pacientes tratados con prótesis reversa por fracturas complejas. El objetivo secundario fue determinar la relación entre rangos de movilidad y puntaje ASES con la evolución radiográfica del troquíter. Materiales y Métodos: Se incluyeron 16 pacientes $>65$ años, tratados con prótesis reversa y reinserción del troquíter, entre 2013 y 2017, operados antes de las 4 semanas del trauma y con un seguimiento mínimo de 2 años. Se consignaron el puntaje ASES y el rango de movilidad activa. En las radiografías, se evaluaron la posición y la consolidación del troquíter, y se registraron las complicaciones y su tratamiento. Resultados: La media de la edad fue 74.5 años (RIC 66-78.5), 11 (69\%) eran mujeres. Once fracturas $(69 \%)$ eran a 4 fragmentos y 5 , luxofracturas a 4 fragmentos. La media entre el trauma y la cirugía fue 9.4 días y el seguimiento, 29.5 meses. En 9 casos (56\%), el troquíter presentó consolidación. Rotación interna: 5 pacientes alcanzaron la región glútea con el pulgar; 4, la vértebra T12; 4, la vértebra L3; 3, la T7. Las medianas de rotación externa y flexión anterior fueron $30^{\circ}$ (RIC 17,5-40) y $100^{\circ}$ (RIC 87,5-160). El puntaje ASES promedio fue 78,3 (RIC 63,3-87,4). No hubo una asociación estadísticamente significativa entre la evolución del troquíter y la flexión anterior y el puntaje ( $p=0,24$ y 0,52 , respectivamente). Conclusión: La prótesis reversa en fracturas agudas con reinserción de las tuberosidades puede llevar a buenos resultados funcionales. No se encontró relación entre la consolidación del troquíter y el puntaje ASES.

Palabras clave: Prótesis reversa; húmero proximal; fracturas; tuberosidades; resultados funcionales; reemplazo de hombro. Nivel de Evidencia: IV (Serie de casos)
\end{abstract}

\section{Functional and radiologic outcomes of reverse shoulder arthroplasty and greater tuberosity} reattachment in proximal humerus fractures

\section{ABSTRACT}

Objective: To report functional and radiologic outcomes of reverse shoulder arthroplasty (RSA) in patients with complex proximal humeral fractures. A second objective was to assess the relation between the greater tuberosity healing and the range of motion (ROM) and the American Shoulder and Elbow Surgeons (ASES) score. Materials and Methods: Sixteen patients treated between 2013 and 2017, older than 65 years old, operated before 4 weeks after the trauma, and with a minimum of 2-year follow-up were included. ASES scores and active ROMs were recorded. Greater tuberosity and the prosthesis position and healing were radiologically evaluated, and the complications and treatment were recorded. Results: The median age was of 74.5 years (IQR 66-78.5), 11 patients were females $(69 \%)$. According to Neer classification, 11 cases were four-part fractures and 5 were four-part fracture-dislocations. The average time between trauma and surgery was 9.4 days, and the average follow-up was of 29.5 months. The greater tuberosity was healed in 9 cases (56\%). Internal rotation: 5 patients $(31.25 \%)$ were able to reach up with their thumbs to gluteal level, $4(25 \%)$ to T12, $3(18.75 \%)$ to T7, and $4(25 \%)$ to L3. The medians for external rotation and forward flexion were $30^{\circ}\left(\right.$ IQR $\left.17.5^{\circ}-40^{\circ}\right)$ and $100^{\circ}$ (IQR $\left.87.5^{\circ}-160^{\circ}\right)$. The average ASES score was of 78.3 (IQR 63.3-87.4). There was no significant statistical relation between greater tuberosity healing and forward flexion or ASES score $(P=0.24$ and $P=0.52$, respectively). Conclusion: The use of reverse prostheses for complex fractures with greater tuberosity reattachment could lead to good functional outcomes, low complication rates and reoperations. There was no significant statistical relation between ASES score and greater tuberosity healing or failure to heal.

Key words: Reverse prosthesis; proximal humerus; fractures; tuberosities; functional outcomes; shoulder arthroplasty.

Level of Evidence: IV (Case series)

Recibido el 30-5-2019. Aceptado luego de la evaluación el 17-10-2019 • Dr. JUAN MARTíN PATIÑO • drpatinojm @ gmail.com

Cómo citar este artículo: Patiño JM, Martínez CE, Andres B, López Bustos F, Ruiz C, Fiminella H, Ferro J, Garay A. Resultados funcionales y radiográficos de la prótesis reversa y la reinserción del troquíter en pacientes con fracturas de húmero proximal. Rev Asoc Argent Ortop Traumatol 2020;85(2):133-138. https://doi.org/ 10.15417/issn.1852-7434.2020.85.2.994 


\section{INTRODUCCIÓN}

Las opciones de tratamiento en pacientes con fracturas y luxofracturas complejas de húmero proximal son el tratamiento incruento, las osteosíntesis, las prótesis parciales o totales anatómicas y las prótesis totales reversas. Según algunos estudios estadísticos, ${ }^{1}$ durante los últimos años, la indicación de prótesis total reversa se ha incrementado en pacientes $>65$ años. Inicialmente el fundamento del uso de este tipo de prótesis fue aprovechar sus características para aspirar a mejores resultados en el rango de movilidad, sin depender de la fijación y la consolidación de las tuberosidades, y lograr un resultado previsible en cuanto a la flexión anterior a expensas del deltoides. Pero estudios posteriores mostraron mejores resultados en la movilidad, principalmente rotaciones, y menos complicaciones por inestabilidad anterior en las series con reparación de las tuberosidades., ${ }^{2,3}$

El objetivo de este estudio fue comunicar los resultados funcionales y radiográficos de pacientes con fracturas complejas (fracturas y luxofracturas a 4 fragmentos, según Neer) de húmero proximal tratados con prótesis reversa y reinserción del troquíter. Como objetivo secundario, se evaluó la asociación entre los rangos de movilidad y el puntaje de la ASES (American Society of Shoulder and Elbow Surgeons) en relación con la evolución radiográfica del troquíter (consolidación vs. sin consolidación).

\section{MATERIALES Y MÉTODOS}

En esta evaluación retrospectiva, se incluyó a pacientes $>65$ años, tratados con prótesis reversa y reinserción del troquíter por fracturas y luxofracturas complejas de húmero proximal, según Neer, operados entre 2013 y 2017, antes de las cuatro semanas del trauma, con un seguimiento mínimo de dos años. Se excluyó a aquellos con secuelas traumáticas, como complicaciones de osteosíntesis previa, seudoartrosis y conversiones de hemiartroplastias a prótesis reversa. Todos fueron operados por tres de los autores (CEM, BA, JMP), cirujanos especialistas, y evaluados por el mismo equipo. Se citó a 19 pacientes, por vía telefónica, y concurrieron 16 de ellos. Se usó el puntaje de la ASES para hombro y codo, se midió la movilidad activa del hombro afectado: flexión anterior con la espalda apoyada en la pared, rotación externa con el brazo en aducción y el codo pegado al tronco, rotación interna con el nivel más alto del pulgar a la espalda. Estas determinaciones se realizaron con goniómetro manual. En la cita para la evaluación final, se solicitaron radiografías de frente y de perfil, en las que se evaluó el troquíter, y se clasificó en: consolidación evidente, reabsorción o migración. Luego, los casos fueron divididos en dos grupos para su análisis: consolidación y sin consolidación. Además, se consignó el tipo de prótesis utilizado, si había aflojamiento del tallo o glena y la presencia o no de muescas óseas en la glena (notching glenoideo). Se registraron también las complicaciones y su eventual tratamiento.

En todos los casos, la reinserción del troquíter y del subescapular se realizó con suturas dobles de alta resistencia mediante puntos transversales pasando por medial al componente humeral y puntos verticales tomando dichas estructuras y perforaciones en el húmero. Además, se usó injerto autólogo de esponjosa metafisario tomado de la cabeza del húmero.

Las variables continuas se comunican como medias y desviación estándar (DE) y medianas y rangos intercuartílicos (RIC), según su distribución, mientras que las categóricas se expresan como frecuencias absolutas y porcentajes. Asimismo, se evaluó la asociación, de manera bivariada, entre los grados de movilidad y el puntaje de la ASES en relación con la evaluación radiográfica del troquíter (consolidación vs. sin consolidación). Para evaluar dicha asociación se utilizaron pruebas paramétricas (test de t Student) o no paramétricas (test de Wilcoxon), acorde a su distribución. Se utilizó el programa STATA versión 13.

Este estudio fue aprobado por el Comité de Ética Institucional (2019-01).

\section{RESULTADOS}

Se evaluó a 16 pacientes. La mediana de la edad fue de 74.5 años (RIC 66-78,5), 11 (69\%) eran mujeres. Once de las 16 fracturas $(69 \%)$ correspondieron a tipo 4 fragmentos, según la clasificación de Neer, mientras que las restantes cinco fueron luxofracturas a 4 fragmentos. La media del tiempo entre el trauma y la cirugía fue de 9.4 días (DE 3), mientras que la media de seguimiento fue de 29.5 meses (DE 7) (Tabla 1). Con respecto a los tipos de prótesis utilizadas, nueve (56\%) eran tallos cementados y, en 10 casos (62,5\%), se utilizaron glenosferas con dos tornillos con quilla central (Arrow, FH Orthopedics, Mulhouse, Francia) y, en los restantes, cuatro tornillos y tetón central (6 Comprehensive ${ }^{\circledR}$ Reverse Shoulder, Zimmer Biomet, Warsaw, IN, EE.UU., 1 Humelock, FX Solutions, Lyon, Francia). En todos los casos, se usaron tallos diseñados para fracturas. 
Tabla 1. Características demográficas y clínicas de los pacientes incluidos

\begin{tabular}{|l|c|}
\hline Variable & Valor \\
\hline Edad, mediana (RIC) & $74,5(66-78,5)$ \\
\hline Sexo femenino, n (\%) & $11(69)$ \\
\hline Tipo de fractura*, n (\%) & $11(69)$ \\
\hline Fractura a 4 fragmentos & $5(31)$ \\
\hline Luxofractura a 4 fragmentos & $9,4 \pm 3$ \\
\hline Tiempo transcurrido hasta la cirugía, media \pm DE
\end{tabular}

$\mathrm{RIC}=$ rango intercuartílico, $\mathrm{DE}=$ desviación estándar. *Según la clasificación de Neer.

En esta serie, las radiografías posoperatorias revelaron que, en nueve pacientes (56\%), el troquíter tenía una adecuada consolidación. Cinco de los siete restantes evolucionaron con reabsorción, un caso requirió la colocación de un espaciador a causa de una infección posoperatoria y uno presentó migración. La evaluación clínica de la rotación interna mostró que cinco pacientes $(31,25 \%)$ alcanzaron la región glútea; cuatro $(25 \%)$, la vértebra T12; cuatro (25\%), la vértebra L3; tres $(18,75 \%)$, T7. Las medianas de la rotación externa y la flexión fueron $30^{\circ}$ (RIC 17,5-40) y $100^{\circ}$ (RIC 87,5-160). Por último, la mediana del puntaje de la ASES fue 78,3 (RIC 63,3-87,4). No se observaron casos de aflojamiento ni muescas en la glena.

Dos pacientes $(12,5 \%)$ tuvieron complicaciones y debieron ser operados nuevamente. Un caso de disociación de la glenosfera que fue resuelto mediante cirugía, a las dos semanas y tuvo una evolución favorable. Un paciente con infección aguda al que se le realizó una limpieza y se le administró tratamiento antibiótico; el paciente evolucionó con control de la infección por ocho meses, pero luego se reagudizó y fue necesario colocarle un espaciador de cemento con antibióticos. El paciente no aceptó la revisión. Finalmente, se llevó a cabo la evaluación de la posible asociación entre el puntaje de la ASES 78,3 (RIC 63,3-87,4) y la presencia de consolidación en la evaluación radiográfica posoperatoria, y el ángulo de flexión en relación con esta misma evolución. En ambos casos, no se halló asociación estadísticamente significativa ( $\mathrm{p}=0,24$ y 0,52 , respectivamente) (Tabla 2, Figura).

Tabla 2. Resultados

\begin{tabular}{|l|c|}
\hline Resultado & Valor \\
\hline Consolidación, n (\%) & $9(56)$ \\
\hline Rotación interna, n (\%) & $5(31,25)$ \\
\hline Región glútea & $4(25)$ \\
\hline T12 & $3(18,75)$ \\
\hline T7 & $3(18,75)$ \\
\hline L3 & $1(6,25)$ \\
\hline Región lumbar & $30^{\circ}\left(17,5-40^{\circ}\right)$ \\
\hline Rotación externa, mediana (RIC) & $100^{\circ}\left(87,5-160^{\circ}\right)$ \\
\hline Flexión, mediana (RIC) & $78,3(63,3-87,4)$ \\
\hline Puntaje de la ASES, mediana (RIC)
\end{tabular}

\footnotetext{
RIC = rango intercuartílico.
} 


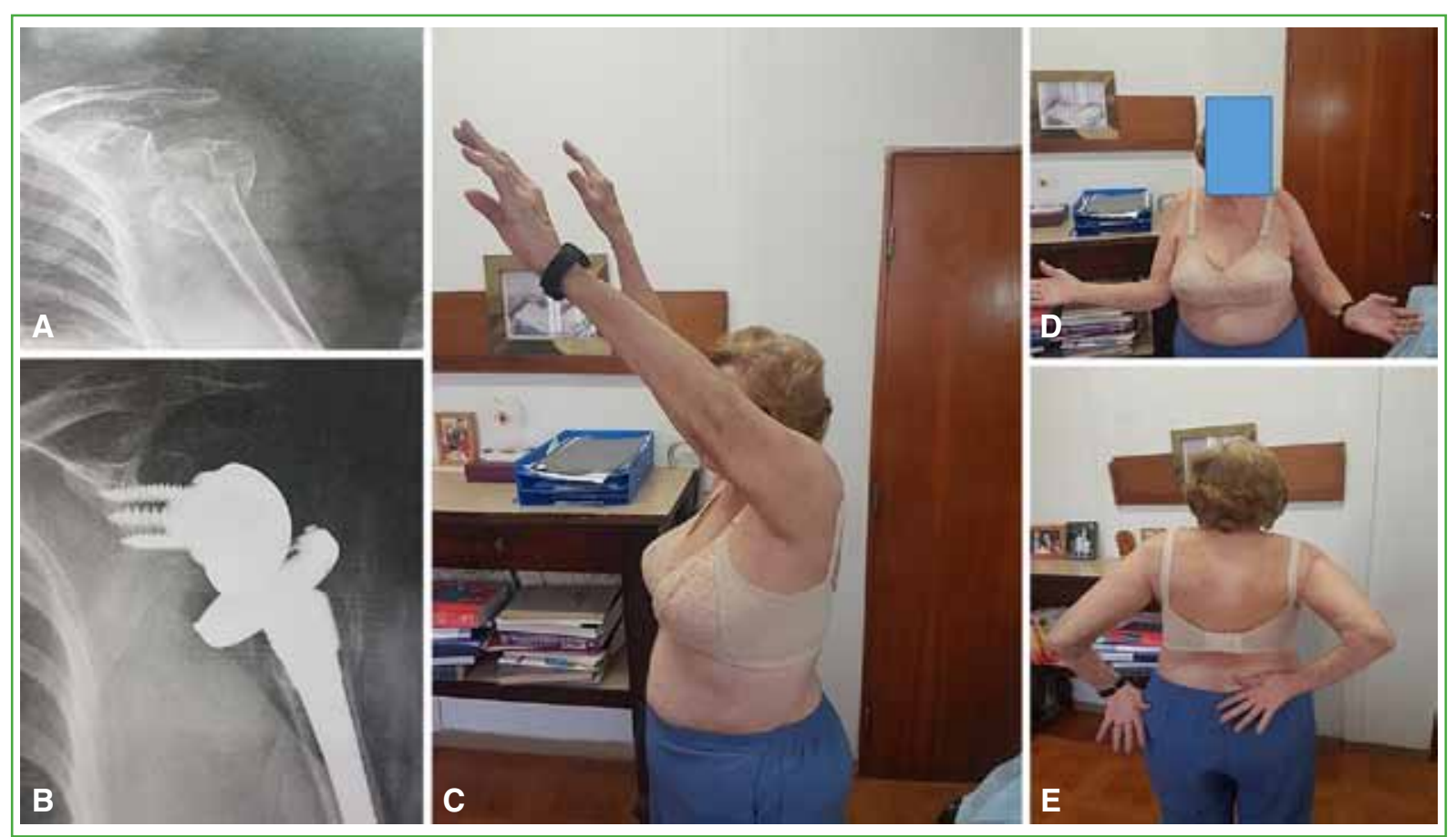

Figura. Mujer de 79 años. A. Radiografía anteroposterior preoperatoria. B. Radiografía anteroposterior a los 30 meses de la cirugía. C-E. Flexión anterior, rotación externa y rotación interna en la evaluación final.

\section{DISCUSIÓN}

En esta serie, se presentan los resultados funcionales y radiográficos de 16 pacientes con fracturas complejas que no se pueden tratar con osteosíntesis, es decir, fracturas y luxofracturas a 4 fragmentos con trazos a nivel del cuello anatómico del húmero, tratadas con prótesis reversa y reinserción del troquíter. Aún no están claros la recomendación y los beneficios de reinsertar, en forma segura, las tuberosidades. Los datos sobre este punto son relativamente recientes y no abunda la bibliografía con resultados funcionales y radiográficos. En la revisión bibliográfica, no hemos encontrado reportes sobre este tema, en nuestro medio.

Se investigó la evolución del troquíter reinsertado, en cuanto a consolidación y funcionalidad del hombro en la particular población de pacientes $>65$ años.

El porcentaje de consolidación del troquíter de nuestra serie (56\%) resultó más alto que el de algunos estudios en los que se han reportado bajas tasas en pacientes mayores (40\%). ${ }^{2-6}$ Se aproximó al de otros autores que llevaron a cabo estudios de similar nivel de evidencia, que lograron alrededor de un $64 \%,{ }^{7,8}$ pero otros autores han comunicado tasas del $80-100 \%{ }^{4,9,10}$

Los tallos diseñados para fracturas, ${ }^{4,10}$ como los usados en nuestra serie, tienen un perfil menor que permite una mejor reinserción y más anatómica con mayor espacio para el uso de injerto de esponjosa. Ambos detalles son importantes para lograr un mejor arco de movilidad., ${ }^{40-12}$

No hallamos una relación estadísticamente significativa entre un puntaje de la ASES más alto y mayor flexión anterior con los casos de consolidación o no del troquíter. En la bibliografía, los casos con migración, reabsorción o seudoartrosis del troquíter se relacionan con menores rangos y más dificultades en actividades de la vida diaria. ${ }^{13-17}$ Es factible que, en esta serie, no se haya comprobado esta relación por el tamaño de la muestra. Por otra parte, no resulta claro qué ocurre funcionalmente con los tendones cuando parte del troquíter se observa reabsorbido. Creemos que, aunque no es evidente en las radiografías, la reabsorción puede ser parcial con remanente de inserciones.

Dentro de los hallazgos podemos citar que la consolidación fue del 56\%; la flexión anterior promedio, de $100^{\circ}$; la rotación externa, de $30^{\circ}$, cifras similares a las de otras series. ${ }^{5,13,14}$

No se detectaron casos de inestabilidad anterior. La reinserción del subescapular puede disminuir esta compli-

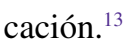


Se han reportado tasas más altas de complicaciones con la no reinserción del troquíter, como inestabilidades, aflojamientos, infecciones y lisis del húmero proximal. ${ }^{15-18}$ Un paciente sufrió una infección y no hubo luxaciones. Creemos que esta última complicación es menos esperable con una adecuada reconstrucción y consolidación del troquíter y la reinserción del subescapular. Un paciente fue operado nuevamente por desacople de la glenosfera, hecho que se relacionó con un error técnico, pero no con inestabilidad.

Boileau y cols., ${ }^{17}$ en un estudio de 38 pacientes, obtuvieron mejores resultados en cuanto a movilidad, infección, inestabilidad y aflojamientos, en los 32 casos con consolidación de las tuberosidades. Los autores teorizan tres ventajas con la reinserción de tuberosidades: primero, el riesgo de potencial inestabilidad es menor por la reconstrucción de la contención anterior y posterior, así como la altura del húmero; segundo, el riesgo de infección es más bajo por la disminución del espacio muerto periprotésico debido a la mayor cantidad de tejido vascularizado óseo y blando. Y, tercero, el riesgo de aflojamiento sería menor, ya que el componente humeral no depende solo de la fijación distal.

Creemos que existen factores locales, como irrigación de los fragmentos óseos y lesiones previas de los músculos rotadores del hombro, que pueden influir en la consolidación de las tuberosidades, además de la técnica de fijación utilizada. Se necesitan estudios sobre estos factores y el desarrollo de las técnicas quirúrgicas para mejorar los resultados y predecir la evolución en cada caso particular.

Las limitaciones de este estudio son las relacionadas con un estudio retrospectivo y el tamaño de la muestra, además de la evaluación con radiografías simples del troquíter únicamente. Consideramos que sus ventajas son el minucioso seguimiento con atención a la funcionalidad y las imágenes radiográficas en una población bien definida >65 años y un homogéneo tipo de fracturas agudas, con un seguimiento mínimo de dos años, junto con el empleo de una técnica similar de reinserción y uso de injerto.

\section{CONCLUSIONES}

En esta serie, el uso de una prótesis reversa en pacientes con fracturas agudas complejas de húmero proximal cuidando la reinserción de las tuberosidades logró buenos resultados funcionales, con una tasa baja de complicaciones y segundas operaciones. No se halló una asociación estadísticamente significativa entre la consolidación o no del troquíter y el puntaje de la ASES.

Conflictos de interés: Los autores no declaran conflictos de intereses.

ORCID de C. E. Martínez: http://orcid org/0000-0002-6031-0532

ORCID de B. Andres: http://orcid.org/0000-0001-7426-5990

ORCID de F. López Bustos: http://orcid.org/0000-0002-2504-2026

ORCID de C. Ruiz: http://orcid.org/0000-0002-3300-0141
ORCID de H. Fiminella: http://orcid.org/0000-0002-7944-2770

ORCID de J. Ferro: https://orcid.org/0000-0003-3720-7884

ORCID de A. Garay: http://orcid.org/0000-0001-6371-0884

\section{BIBLIOGRAFÍA}

1. Rosas S, Law TY, Kurowicki J, Formaini N, Kalandiak SP, Levy JC. Trends in surgical management of proximal humeral fractures in the Medicare population: a nationwide study of records from 2009 to 2012. J Shoulder Elbow Surg 2016;25(4):608-13. https://doi.org/10.1016/j.jse.2015.08.011

2. Bufquin T, Hersan A, Hubert L, Massin P. Reverse shoulder arthroplasty for the treatment of three-and four-part fractures of the proximal humerus in the elderly: a prospective review of 43 cases with a short-term follow-up. $J$ Bone Joint Surg Br 2007;89(4):516-20. https://dx.doi.org/10.1302/0301-620X.89B4.18435

3. Chun YM, Kim DS, Lee DH, Shin SJ. Reverse shoulder arthroplasty for four-part proximal humerus fracture in elderly patients: can a healed tuberosity improve the functional outcomes? J Shoulder Elbow Surg 2017;26(7):121621. https://dx.doi.org/10.1016/j.jse.2016.11.03410.1302/0301-620X.89B4.18435

4. Garofalo R, Flanagin B, Castagna A, Lo EY, Krishnan SG. Reverse shoulder arthroplasty for proximal humerus fracture using a dedicated stem: radiological outcomes at a minimum 2 years of follow-up—case series. J Orthop Surg Res 2015;10:129. https://dx.doi.org/10.1186/s13018-015-0261-1 
5. Grubhofer F, Wieser K, Meyer DC, Catanzaro S, Beeler S, Riede U, et al. Reverse total shoulder arthroplasty for acute head-splitting, 3- and 4-part fractures of the proximal humerus in the elderly. J Shoulder Elbow Surg 2016;25(10):1690-8. https://dx.doi.org/10.1016/j.jse.2016.02.024

6. Uzer G, Yildiz F, Batar S, Binlaksar R, Elmadag M, Kus G, et al. Does grafting of the tuberosities improve the functional outcomes of proximal humeral fractures treated with reverse shoulder arthroplasty? J Shoulder Elbow Surg 2017;26(1):36-41. http://dx.doi.org/10.1016/j.jse.2016.05.005

7. Boileau P, Krishnan SG, Tinsi L,Walch G, Coste JS, Molé D. Tuberosity malposition and migration: reasons for poor outcomes after hemiarthroplasty for displaced fractures of the proximal humerus. J Shoulder Elbow Surg 2002;11(5):401-12. https://doi.org/10.1067/mse.2002.124527

8. Sebastiá-Forcada E, Cebrián-Gómez R, Lizaur-Utrilla A, Gil-Guillén V. Reverse shoulder arthroplasty versus hemiarthroplasty for acute proximal humeral fractures. A blinded, randomized, controlled, prospective study. J Shoulder Elbow Surg 2014;23(10):1419-26. https://dx.doi.org/10.1016/j.jse.2014.06.035

9. Formaini NT, Everding NG, Levy JC, Rosas S. Tuberosity healing after reverse shoulder arthroplasty for acute proximal humerus fractures: the "black and tan" technique. J Shoulder Elbow Surg 2015;24(11):e299-306. https://dx.doi.org/10.1016/j.jse.2015.04.014

10. Levy JC, Badman B. Reverse shoulder prosthesis for acute four-part fracture: tuberosity fixation using a horseshoe graft. J Orthop Trauma 2011;25(5):318-24. https://dx.doi.org/10.1097/BOT.0b013e3181f22088

11. Boileau P, Krishnan SG, Tinsi L, Walch G, Coste JS, Molé D. Tuberosity malposition and migration: reasons for poor outcomes after hemiarthroplasty for displaced fractures of the proximal humerus. J Shoulder Elbow Surg 2002;11(5):401-12. https://dx.doi.org/10.1067/mse.2002.124527

12. Boileau P, Winter M, Cikes A, Han Y, Carles M, Walch G, et al. Can surgeons predict what makes a good hemiarthroplasty for fracture? J Shoulder Elbow Surg 2013;22(11):1495-506. https://dx.doi.org/10.1016/j.jse.2013.04.018

13. Matthewson G, Kooner S, Kwapisz, A, Leiter J, Old J, MacDonald P. The effect of subscapularis repair on dislocation rates in reverse shoulder arthroplasty: a meta-analysis and systematic review. J Shoulder Elbow Surg 2019;28(5):989-97. https://dx.doi.org/10.1016/j.jse.2018.11.069

14. Cazeneuve JF, Cristofari DJ, Charalambous CP, Abiddin Z, Mills SP, Rogers S, et al. The reverse shoulder prosthesis in the treatment of fractures of the proximal humerus in the elderly. J Bone Joint Surg Br 2010;92(4): 535-9. https://dx.doi.org/10.1302/0301-620X.92B4.22450

15. Gallinet D, Adam A, Gasse N, Rochet S, Obert L. Improvement in shoulder rotation in complex shoulder fractures treated by reverse shoulder arthroplasty. J Shoulder Elbow Surg 2013;228(1):38-44. https://dx.doi.org/10.1016/j.jse.2012.03.011

16. Klein M, Juschka M, Hinkenjann B, Scherger B, Ostermann PA. Treatment of comminuted fractures of the proximal humerus in elderly patients with the Delta III reverse shoulder prosthesis. J Orthop Trauma 2008;22(10):698-704. https://dx.doi.org/10.1097/BOT.0b013e31818afe40

17. Boileau P, Alta TD, Decroocq L, Sirveaux F, Clavert P, Favard L, et al. Reverse shoulder arthroplasty for acute fractures in the elderly: is it worth reattaching the tuberosities? J Shoulder Elbow Surg 2019;28(3):437-44. https://dx.doi.org/10.1016/j.jse.2018.08.025

18. Ohl X, Bonnevialle N, Gallinet, D, Ramdane N, Valenti P, Decroocq J, et al. How the greater tuberosity affects clinical outcomes after reverse shoulder arthroplasty for proximal humeral fractures. J Shoulder Elbow Surg 2018;27(12):2139-44. https://dx.doi.org/10.1016/j.jse.2018.05.030 